\title{
A Rare Case of Coil Migration into the Duodenum after Embolization of a Right Colic Artery Pseudoaneurysm
}

\author{
Jeongmin Choi and Young Moon Kim \\ Department of Internal Medicine, Sanggye Paik Hospital, Inje University College of Medicine, Seoul, Korea
}

Transcatheter arterial embolization is a safe and effective treatment for visceral artery aneurysms; nevertheless, some complications can occur. Coil migration to other organs after embolization is extremely rare, and only 16 cases have been reported previously. We report a rare case of coil migration to the duodenal lumen after embolization of a right colic artery pseudoaneurysm. To the best of our knowledge, this is the first case of coil migration after a right colic artery embolization. The patient exhibited no symptoms and was treated conservatively without any intervention. Some previous reports have demonstrated spontaneous coil passage and successful conservative management. Our case supports conservative treatment as the primary treatment for asymptomatic patients. Clinicians should assess the risks and benefits of coil removal in asymptomatic patients before performing any intervention. Clin Endosc 2021;54:920-923

Key Words: Adverse effects; Endoscopy; False aneurysm; Foreign-body migration; Therapeutic embolization

\section{INTRODUCTION}

Visceral artery pseudoaneurysms are rare and can be caused by atherosclerosis, pancreatitis, cholecystitis, inflammation, or trauma. These occur mainly in the splenic and hepatic arteries and rarely in the gastric artery, and they require urgent treatment as the mortality rates following their rupture are as high as $20-40 \% .{ }^{1}$ Transcatheter arterial embolization is usually performed because it is safe and effective. ${ }^{2}$

Complications after coil embolization include re-bleeding, infarction, abscesses, coil migration to other organs, and dissection of puncture vessel. Of these complications, coil migration to other organs after endovascular embolization is

Received: August 19, 2020 Revised: September 24, 2020

Accepted: October 12, 2020

Correspondence: Jeongmin Choi

Department of Internal Medicine, Sanggye Paik Hospital, Inje University College of Medicine, 1342 Dongil-ro, Nowon-gu, Seoul 01757, Korea

Tel: +82-2-950-1001, Fax: +82-2-950-6701, E-mail: doct00@hanmail.net

ORCID: https://orcid.org/0000-0001-6976-9887

(c) This is an Open Access article distributed under the terms of the Creative Commons Attribution Non-Commercial License (http://creativecommons.org/ licenses/by-nc/3.0) which permits unrestricted non-commercial use, distribution, and reproduction in any medium, provided the original work is properly cited. extremely rare, and only 16 cases have been reported previously. ${ }^{3-18}$

We report a rare case of coil migration to the duodenal lumen after transcatheter arterial embolization of a right colic artery pseudoaneurysm. To the best of our knowledge, this is the first reported case of coil migration after embolization of a right colic artery pseudoaneurysm. Finally, we recommend a management approach for asymptomatic patients with coil migration.

\section{CASE REPORT}

A 62-year-old man visited the emergency room with complaints of fever, vomiting, and epigastric pain since 1 week. The patient had hypertension and diabetes. A computed tomography (CT) scan revealed a pseudoaneurysm of the branch of the superior mesenteric artery and an organized hematoma in the distal small bowel (Fig. 1A). Angiography was then performed, showing a $4 \times 3 \mathrm{~cm}$ sized pseudoaneurysm of the right colic artery branch in the superior mesenteric artery (Fig. 1B). No contrast extravasation was observed. However, as the risk of rupture seemed high, we decided to perform embolization. Three coils were used for embolization therapy 

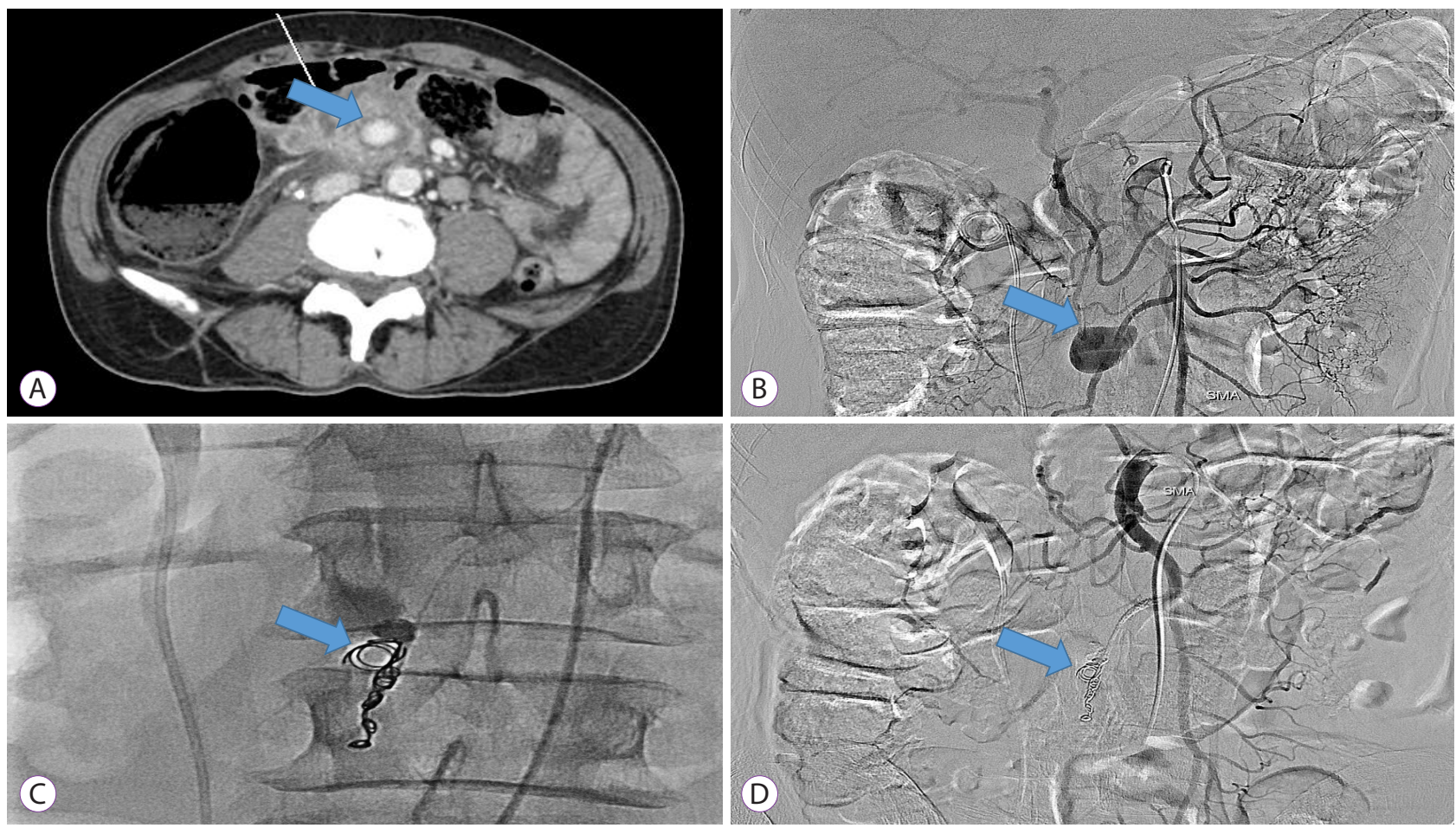

Fig. 1. (A) Computed tomography shows a pseudoaneurysm of the superior mesenteric artery (arrow) and hematoma in the small bowel. (B) Angiography shows a pseudoaneurysm of the branch of the right colic artery (arrow). (C) Embolization of the pseudoaneurysm was performed using coils. (D) Angiographic image after endovascular coiling shows multiple coils (arrow) in the successfully occluded pseudoaneurysm.
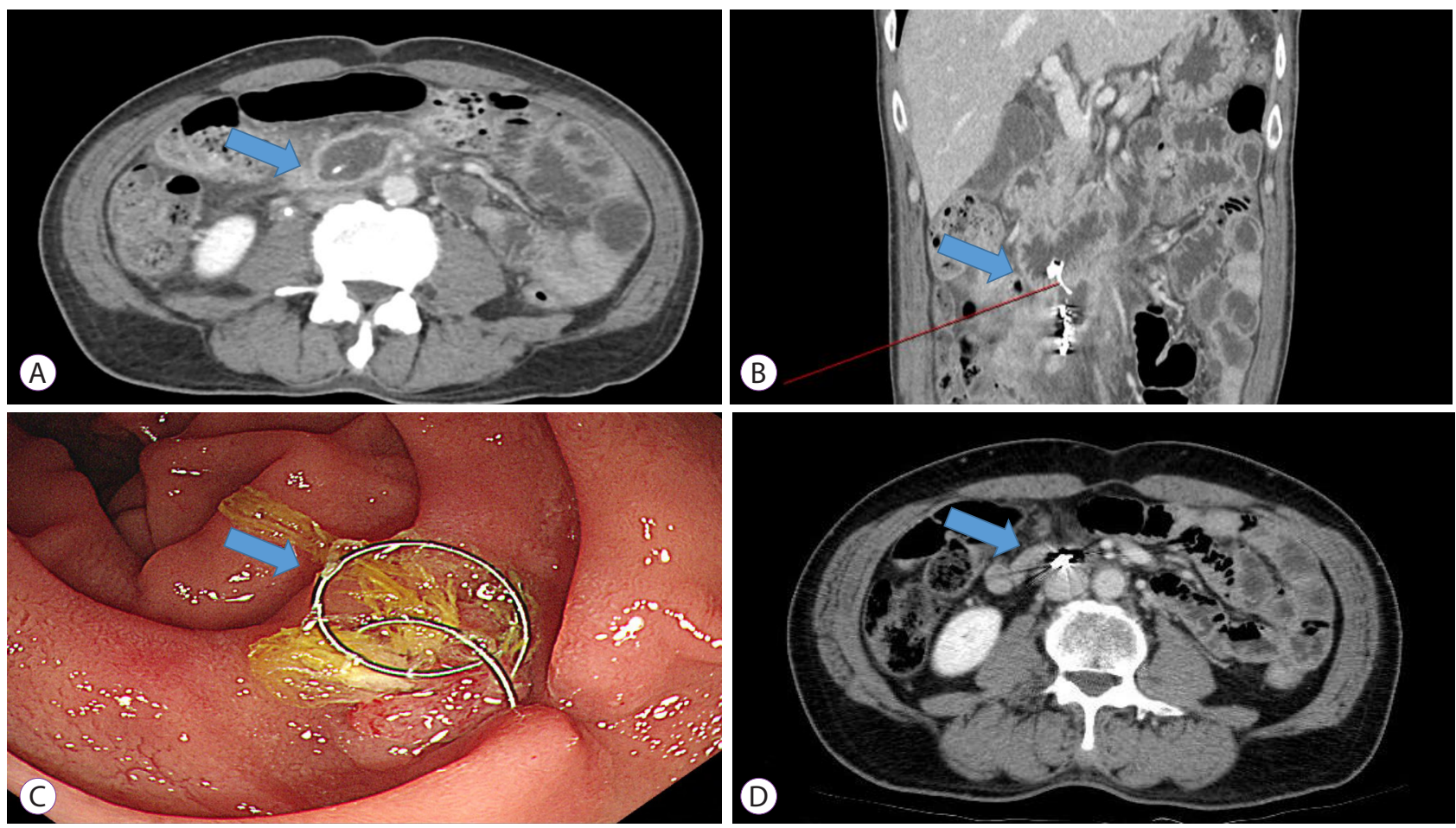

Fig. 2. (A, B) After 6 days of embolization, computed tomography reveals that the coils had migrated to the third wall of the duodenum (arrow). (C) Upper endoscopy shows that two strands of the wire penetrated the third wall of the duodenum (arrow). Surrounding granulation tissue is seen. (D) No interval change is observed at the 2-month follow-up computed tomography (arrow). 
(Fig. 1C). Specifically, embolization coils (Tornado ${ }^{\circledR}$ emboli- $^{-}$ zation coil, $5 \mathrm{~cm}$ long, 0.018-inch diameter; COOK Medical LLC., Bloomington, IN, USA) were deployed at the distal end of the pseudoaneurysm. Next, embolization with a mixture of histoacryl and lipiodol was performed. After the procedure, superior mesenteric artery angiography showed no contrast filling in the pseudoaneurysm, confirming successful embolization (Fig. 1D). The patient's abdominal pain improved, and his vital signs stabilized.

A follow-up abdominal CT six days after embolization showed penetration of coil material into the lumen of the third part of the duodenum (Fig. 2A, B). The patient had no fever, abdominal pain, or melena. He was carefully observed without further intervention and was discharged on the 16th day after embolization.

One month after discharge, an upper gastrointestinal endoscopy was performed to check if the coil had moved into the duodenal wall. It showed two strands of wire pulled out from the lumen inside of the third part of the duodenum that was covered with granulation tissue (Fig. 2C). The surrounding duodenal mucosa was normal, without necrosis or fistula. Initially, we tried to remove the coil using biopsy forceps; however, it was firmly attached to the duodenal wall. We believed it would be difficult and even harmful to remove the coil endoscopically since the coil was spiral-shaped. We decided to adopt a conservative approach by not removing the coil. Two months later, an abdominal CT was performed. The displaced coil was still seen in the duodenal wall (Fig. 2D). After 1 year of follow-up, no complications were observed.

\section{DISCUSSION}

We searched for previous case reports of coil migration after endovascular embolization for visceral artery pseudoaneurysm. Finally, a total of 17 cases, including our case, were reviewed. ${ }^{3-18}$ Most patients had various underlying illnesses, including chronic pancreatitis or cholecystitis. The common sites of pseudoaneurysms were hepatic, splenic, and gastroduodenal arteries. The common migration sites were the stomach and common bile duct. Approximately $70 \%$ of patients showed obstructive signs and symptoms. Patients with coil migration to the common bile duct showed signs of cholangitis or had biliary colic pain.

Although the exact reason for coil migration is unclear, there are few reports about the risk factors associated with coil migration. Several reports mentioned the presence of infection, ${ }^{9}$ malignancy, ${ }^{3}$ or inflammation ${ }^{18}$ around the pseudoaneurysm. Under these conditions, the coil may erode the thin walls of the pseudoaneurysm and migrate to another organ. As the sizes of the pseudoaneurysm varied dramatically, the number and sizes of the coils deployed also varied greatly across cases. The size of the coil used for embolization was found to be unrelated to the chance of its migration. ${ }^{4}$

The duration between coil migration and embolization varied from several weeks to 10 years. In our patient, the migration developed unexpectedly early, i.e., 6 days after embolization. There is a possibility of intra-procedural coil migration. The very thin $(0.05 \mathrm{~mm})$ tip of the endovascular coil may damage the thin wall of the pseudoaneurysm and later penetrate into the adjacent organ. However, endovascular damage by the coil could not be identified in our study since angiography did not show extravasation after the embolization. In one study, an intra-procedural complication was reported, including rupture of the gastroduodenal artery by an endovascular coil. $^{19}$

Treatment for migrated coils has not been established yet, owing to the small number of cases. Among the 17 patients with coil migration, patients underwent endoscopic removal $(n=4)$, surgical exploration $(n=6)$ or combined endoscopic surgery $(n=1)$, and conservative treatment $(n=5)$. The remaining 1 patient had a fatal aortogastric fistula as a remote complication of embolization.

Patients whose coils migrated to the common bile duct underwent endoscopic retrograde cholangioscopic removal of the migrated coils, ${ }^{7,11}$ percutaneous cholangioscopic extraction, ${ }^{15}$ or open surgical exploration. ${ }^{16,17}$ One patient with coil migration to the jejunum underwent upper endoscopic removal. ${ }^{8}$ Hot biopsy forceps were used for the removal of the thick coil wires. ${ }^{8}$ Most patients had good treatment outcomes.

Treating patients with asymptomatic coil migration is challenging because little is known about the long-term course of natural disease. In 3 out of 5 asymptomatic patients, the migrated coils passed spontaneously through the stomach, ${ }^{3}$ rectum, ${ }^{13}$ or ureterovesical junction. ${ }^{12}$ Matsubara et al. ${ }^{3}$ reported on coil migration into the stomach after embolization for splenic artery pseudoaneurysm. The patient was carefully followed-up, because there was no active bleeding or peritonitis, and the coil was excreted spontaneously 6 months later. Our patient had no signs of obstruction, fistula, or any other symptoms. The migrated coil penetrated the duodenal wall that was covered with granulation tissue and a normal surrounding mucosal layer. As it was hard to remove it endoscopically, the patient was followed up and no complications were observed for 12 months.

In conclusion, we reported a rare case of coil migration to the duodenal lumen after embolization of a right colic artery pseudoaneurysm. The patient showed no symptoms and was treated conservatively without any intervention. Our case supports conservative treatment as the primary treatment for 
asymptomatic patients. Clinicians should assess the risks and benefits of coil removal in asymptomatic patients, prior to performing an intervention.

\section{Conflicts of Interest}

The authors have no potential conflicts of interest.

Funding

This work was supported by the 2019 Inje University research grant (No.2019001)

Author Contributions
Conceptualization: Jeongmin Choi, Young Moon Kim
Methodology: JC, YMK
Writing-original draft: JC, YMK
Writing-review\&editing: JC
$\begin{array}{ll}\text { ORCID } & \\ \text { Jeongmin Choi: } & \text { https://orcid.org/0000-0001-6976-9887 } \\ \text { Young Moon Kim: } & \text { https://orcid.org/0000-0001-6056-0905 }\end{array}$

\section{REFERENCES}

1. Pitton MB, Dappa E, Jungmann F, et al. Visceral artery aneurysms: incidence, management, and outcome analysis in a tertiary care center over one decade. Eur Radiol 2015;25:2004-2014.

2. Barrionuevo P, Malas MB, Nejim B, et al. A systematic review and meta-analysis of the management of visceral artery aneurysms. J Vasc Surg 2019;70:1694-1699.

3. Matsubara Y, Lim LA, Hijikata Y, Hirata Y, Yotsuyanagi H. Embolization coil migration in the stomach and spontaneous excretion: a case report and review of the literature. Radiol Case Rep 2020;15:1018-1022.

4. Hewgley WP, Webb DL, Garrett HE Jr. Migrated embolization coil causes intestinal obstruction. J Vasc Surg Cases Innov Tech 2018;4:8-11.

5. Pratap A, Pokala B, Vargas LM, Oleynikov D, Kothari V. Laparoscopic endoscopic combined surgery for removal of migrated coil after embolization of ruptured splenic artery aneurysm. J Surg Case Rep 2018;2018:rjx242.

6. Nomura Y, Gotake Y, Okada T, Yamaguchi M, Sugimoto K, Okita Y. Coil migration to the duodenum 1 year following embolisation of a ruptured giant common hepatic artery aneurysm. EJVES Short Rep 2018;39:3336.

7. Zaafouri H, Hasnaoui A, Essghaeir S, et al. Ascending cholangitis secondary to migrated embolization coil of gastroduodenal artery pseudo-aneurysm a case report. BMC Surg 2017;17:30.

8. Han YM, Lee JY, Choi IJ, et al. Endoscopic removal of a migrated coil after embolization of a splenic pseudoaneurysm: a case report. Clin Endosc 2014;47:183-187.

9. Tekola BD, Arner DM, Behm BW. Coil migration after transarterial coil embolization of a splenic artery pseudoaneurysm. Case Rep Gastroenterol 2013;7:487-491.

10. Skipworth JR, Morkane C, Raptis DA, et al. Coil migration--a rare complication of endovascular exclusion of visceral artery pseudoaneurysms and aneurysms. Ann R Coll Surg Engl 2011;93:e19-e23.

11. Kao WY, Chiou YY, Chen TS. Coil migration into the common bile duct after embolization of a hepatic artery pseudoaneurysm. Endoscopy 2011;43(Suppl 2):E364-E365.

12. Reed A, Suri R, Marcovich R. Passage of embolization coil through urinary collecting system one year after embolization. Urology 2007;70:1222.e17-e18.

13. Shah NA, Akingboye A, Haldipur N, Mackinlay JY, Jacob G. Embolization coils migrating and being passed per rectum after embolization of a splenic artery pseudoaneurysm, "the migrating coil": a case report. Cardiovasc Intervent Radiol 2007;30:1259-1262.

14. Dinter DJ, Rexin M, Kaehler G, Neff W. Fatal coil migration into the stomach 10 years after endovascular celiac aneurysm repair. J Vasc Interv Radiol 2007;18(1 Pt 1):117-120.

15. Van Steenbergen W, Lecluyse K, Maleux G, Pirenne J. Successful percutaneous cholangioscopic extraction of vascular coils that had eroded into the bile duct after liver transplantation. Endoscopy 2007;39(Suppl 1):E210-E211.

16. Turaga KK, Amirlak B, Davis RE, Yousef K, Richards A, Fitzgibbons RJ Jr. Cholangitis after coil embolization of an iatrogenic hepatic artery pseudoaneurysm: an unusual case report. Surg Laparosc Endosc Percutan Tech 2006;16:36-38.

17. Ozkan OS, Walser EM, Akinci D, Nealon W, Goodacre B. Guglielmi detachable coil erosion into the common bile duct after embolization of iatrogenic hepatic artery pseudoaneurysm. J Vasc Interv Radiol 2002;13(9 Pt 1):935-938.

18. Takahashi T, Shimada K, Kobayashi N, Kakita A. Migration of steelwire coils into the stomach after transcatheter arterial embolization for a bleeding splenic artery pseudoaneurysm: report of a case. Surg Today 2001;31:458-462.

19. Xu H, Jing C, Zhou J, et al. Clinical efficacy of coil embolization in treating pseudoaneurysm post-whipple operation. Exp Ther Med 2020;20:37. 